\title{
Pseudomonas aeruginosa infection of avian origin: Zoonosis and one health implications
}

\author{
Wafaa A. Abd El-Ghany (i) \\ Department of Poultry Diseases, Faculty of Veterinary Medicine, Cairo University, Egypt. \\ Corresponding author: Wafaa A. Abd El-Ghany, e-mail: wafaa.ghany@yahoo.com \\ Received: 22-05-2021, Accepted: 20-07-2021, Published online: 21-08-2021
}

doi: www.doi.org/10.14202/vetworld.2021.2155-2159 How to cite this article: Abd El-Ghany WA (2021) Pseudomonas aeruginosa infection of avian origin: Zoonosis and one health implications, Veterinary World, 14(8): 2155-2159.

\begin{abstract}
Zoonotic diseases are diseases that are transmitted from animals to humans and vice versa. Pseudomonas aeruginosa ( $P$. aeruginosa) is a pathogen with zoonotic nature. Commercial poultry could be infected with $P$. aeruginosa, especially at young ages with great losses. Infection of embryos with $P$. aeruginosa induced death in the shell, while infection of chicks led to septicemia, respiratory and enteric infections, and high mortality. Humans are also highly susceptible to $P$. aeruginosa infection, and the disease is associated with severe lung damage, especially in immunocompromised patients. Chicken carcass and related poultry retail products play an important role in the transmission of P. aeruginosa to humans, especially after processing in abattoirs. Treatment of P. aeruginosa infection is extremely difficult due to continuous development of antibiotic resistance. The transfer of antibiotic-resistant genes from poultry products to humans creates an additional public health problem. Accordingly, this study focused on avian pseudomonad, especially P. aeruginosa, with respect to infection of poultry, transmission to humans, and treatment and antibiotic resistance.
\end{abstract}

Keywords: antibiotic resistance, human, poultry, Pseudomonas aeruginosa, zoonosis.

\section{Introduction}

The World Health Organization [1] defined any infection that is transmitted naturally from animals to humans or from humans to animals as zoonosis. Approximately 2.4 billion diseased cases and 2.7 million deaths in humans annually, along with the negative impact on livestock production, are related to these zoonotic infections, especially in low-income countries [2]. Most infectious and fatal diseases that infect humans are of animal or animal product origin [3]. There is a wide variety of bacterial zoonosis in poultry; among them, Pseudomonas aeruginosa $(P$. aeruginosa) infection has a direct public health hazard.

The significance of this study focuses on the problem of avian $P$. aeruginosa infection and its association with human health. $P$. aeruginosa is a ubiquitous organism that often presents in soil, water, and humid environments [4]. The bacterium can colonize soil and infect aquatic habitats, animals, and plants [5]. Moreover, $P$. aeruginosa is regarded as a normal inhabitant and opportunistic organism of avian spp. under normal environmental conditions. However, under stressors, such as immunosuppression, this organism becomes pathogenic and induces a clinical picture. Infection with $P$. aeruginosa in birds is associated with septicemia, respiratory signs, diarrhea, and

\footnotetext{
Copyright: Abd El-Ghany. Open Access. This article is distributed under the terms of the Creative Commons Attribution 4.0 International License (http://creativecommons.org/licenses/ by/4.0/), which permits unrestricted use, distribution, and reproduction in any medium, provided you give appropriate credit to the original author(s) and the source, provide a link to the Creative Commons license, and indicate if changes were made. The Creative Commons Public Domain Dedication waiver (http:// creativecommons.org/publicdomain/zero/1.0/) applies to the data made available in this article, unless otherwise stated.
}

deaths [6], with serious economic losses in the poultry industry [7]. The pathogen has been associated with high mortality in young chickens and late death in the shell of embryos [8].

In processing poultry plants, Pseudomonas spp. have been the predominant organisms on chicken carcasses $[9,10]$. Retail chicken products are incriminated as a primary source of Pseudomonas spp. for humans. Infection with $P$. aeruginosa induces severe pulmonary infection [11] and cystic lung fibrosis [12], especially in immunosuppressed persons.

In many countries, some antibiotics that are used in animal production could serve as essential medications for humans $[13,14]$. Due to the misuse of antibiotics in the animal-food production system, the global surge and spread of antibiotic resistance have been developed $[15,16]$. The food chain has been found to be an important reservoir of antibiotic-resistant Pseudomonas spp. [17]. Unfortunately, the rate of antibiotic resistance of Pseudomonas spp. in the food chain has been increasing worldwide [18].

Accordingly, this review article aimed to focus on avian pseudomonad, especially $P$. aeruginosa, with respect to infection of poultry, transmission to humans, and treatment and antibiotic resistance.

\section{Pathogen}

Among pseudomonad, $P$. aeruginosa is the most common spp. causing poultry and human infections. Isolation and identification of $P$. aeruginosa revealed that the bacterium is Gram-negative, motile, non-spore former, and non-capsulated aerobic bacillus [19]. Isolates of $P$. aeruginosa can grow under aerobic conditions, producing $\beta$-zone of hemolysis on blood agar and non-lactose fermenter colonies on 
MacConkey agar. On the selective Pseudomonas agar base, the organism produces greenish pigment that is characterized by fruity smell. Serological examination of $P$. aeruginosa revealed that the most predominant serotypes were A, B, D, F, H, K, L, and M [20]. False-negative culture results due to overgrowth by other bacteria or presence of non-cultivable or mutant organisms induce some difficulties in using usual old techniques for detection of $P$. aeruginosa [21]. Qin et al. [22] recorded that the identification of $P$. aeruginosa with conventional methods takes a long time to perform and requires extensive hands-on work by technicians. Therefore, recent molecular methods have been developed for an accurate, specific, sensitive, and rapid diagnosis of the pathogen $[23,24]$. Molecular techniques, such as polymerase chain reaction (PCR), are used for the detection of $P$. aeruginosa DNA. In the study of Shahat et al. [25], the use of PCR with 16Sr DNA primers at 956 bp confirmed the existence of $P$. aeruginosa DNA in seven isolates collected from dead-in-shell embryos and broiler chickens in Egyptian poultry farms.

Several studies revealed the ability of $P$. aeruginosa strains to induce high mortalities, septicemic picture, un-absorbed yolk sac, pneumonia, necrosis of different organs, and enteritis in inoculated chicks [24,26,27]. Different virulent factors as tox $A$, psIA, and fliC genes can induce toxicity and pathogenicity of $P$. aeruginosa [24,28,29]. These factors inhibit protein biosynthesis, form biofilms, and have essential roles in the organism's colonization and penetration of cells along with induction of necrosis and death of tissues [30-32]. The mechanism of P. aeruginosa infection has been studied by Li et al. [33]. After adherence and colonization of $P$. aeruginosa to the epithelium of the respiratory tract, macrophages become active, secrete different inflammatory factors, and activate the NF- $\mathrm{KB}$ pathway.

\section{Poultry infection}

Birds of all ages are susceptible to $P$. aeruginosa infection, but young birds are severely affected. Birds can be infected with $P$. aeruginosa by a mechanical route through skin injury or use of contaminated needles during the vaccination process. Several factors, including the bird's immune status and presence of other concomitant infections, may enhance the susceptibility to $P$. aeruginosa infection [6]. Infection with virulent strains of $P$. aeruginosa, especially in newly hatched chicks, resulted in dyspnea, diarrhea, dehydration, septicemia, and high mortality [24,34].

Different surveillance studies have been conducted to detect the incidence of $P$. aeruginosa infection among different chicken flocks. For instance, Satish and Priti [35] isolated $P$. aeruginosa at a rate of $12 \%$ from healthy chicks and $30 \%$ from diseased ones. Furthermore, $P$. aeruginosa was recovered in an incidence rate of $21.6 \%$ [36], 8.7\% [26], and $17.6 \%$ [26] from broiler chicken flocks in different
Egyptian governorates. Conversely, Hassan [37] isolated $P$. aeruginosa from diseased and dead broiler and day-old chicks in incidences of 25.3 and $10 \%$, respectively. Moreover, $P$. aeruginosa has been detected in 42 of $480(8.75 \%)$ broiler chicken samples [38] and 26 of $50(52 \%)$ of dead-in-shell embryos [39]. Recently, 32 of 46 broiler chicken farms (69.57\%) and 183 of 460 chicks $(39.78 \%)$ were positive for $P$. aeruginosa [40]. Eraky et al. [24] recovered $P$. aeruginosa from $16(8 \%)$ of 200 hatcheries and 17 (8.25\%) of 206 chicken embryos samples.

\section{Human infection}

$P$. aeruginosa is regarded as one of the most important pathogens that cause human opportunistic infections [41]. Besides, P. aeruginosa is considered a relevant and most frequently found pathogen causing severe acute nosocomial infections, especially in immunocompromised persons or patients in the intensive care unit [42]. After immunosuppression, the pathogen can cause many secondary infections, including chronic pulmonary lesions [11], cystic fibrosis of the lung, and other chronic underlying diseases [43].

Many studies indicated infection of humans with Pseudomonas spp. through occupational contact with poultry carcasses or related products. Pseudomonas spp. are regarded as important spoilage organisms that are present in spoiled poultry meat sold in retail settings $[44,45]$. Poultry processing plants are considered a potential source of human infection with Pseudomonas. After processing chicken carcasses, some Pseudomonas spp. in processed products were found viable and multiplied under cold air and water, as well as aerobic storage [44]. It has been documented that Pseudomonas spp. can spoil chicken's carcass meat through production of proteolytic, saccharolytic, lipolytic, and biosurfactant changes, especially at the end of shelf life [45]. The predominance and persistence of Pseudomonas spp. in foods and on food processing surfaces may be related to the ability of these microorganisms to form a biofilm, which enhances their tolerance to harsh adverse conditions, including antimicrobial treatments $[46,47]$.

\section{Treatment and antibiotic resistance}

The sensitivity of $P$. aeruginosa strains to different antibiotic classes is extremely variable. For example, $P$. aeruginosa showed sensitivity to ciprofloxacin and gentamycin [39]. Moreover, sensitivity testing of $P$. aeruginosa strains of chicken origin showed susceptibility to levofloxacin, enrofloxacin, and danofloxacin in percentages of $81.25 \%, 59.375 \%$, and $46.875 \%$, respectively, while they showed complete resistance to nalidixic acid (100\%) [40]. Egyptian chicken strains of $P$. aeruginosa showed $100 \%$ susceptibility to ciprofloxacin and norfloxacin, but $100 \%$ resistance to sulfamethazine, erythromycin, ampicillin, tetracycline, amoxicillin, and erythromycin [25]. Besides, Eraky et al. [24] demonstrated that all 
isolated $P$. aeruginosa strains $(100 \%)$ were sensitive to ciprofloxacin, levofloxacin, and gentamycin but resistant $(100 \%)$ to amoxicillin/clavulanic acid, doxycycline, and erythromycin.

Recently, the role of poultry products as a source of human foodborne pathogens, along with the transmission of antibiotic-resistant genes to humans, has emerged $[16,48]$. The presence of active pharmaceutical compounds from antibiotic manufacturing plants and serious pollutants of water and environment participated in the selection of antibiotic-resistant pathogens that pose a significant threat to the public [49].

Multidrug resistance to different antibiotic classes among $P$. aeruginosa isolates has been defined [50,51]. For instance, strains of $P$. aeruginosa that were isolated from diseased and dead chickens in Egypt showed multiple resistance to ampicillin, lincomycin, nalidixic acid, trimethoprim-sulfamethoxazole, chloramphenicol, tetracycline, and doxycycline [38]. Recently, the antibiotic resistance profile of $P$. aeruginosa isolates in poultry abattoirs in Nigeria showed a high level of resistance to carbapenems and cephalosporins [52]. These antibiotics have been employed in poultry production and human treatment [14].

High resistance of $P$. aeruginosa to most antibiotics may be relevant to the low permeability of the bacterial outer membrane lipoprotein (oprL gene) that is implicated in multidrug efflux transport system pump of RND-MFD-OMF encoded in its genome [53]. Furthermore, $P$. aeruginosa has various virulence factors, such as lipopolysaccharide, elastase, alkaline proteases, pyocyanin, pyoverdine, hemolysins, phospholipase $\mathrm{C}$, and rhamnolipids. These factors are coordinated by a global regulatory system, which is activated by autoinducers involving lasI gene [54]. Moreover, exoS, exoT, exoU, and exo $Y$ genes of $P$. aeruginosa, which are important for production of toxic effector proteins into the cytosol of host cells, have been demonstrated.

Detection of some efflux pumps system genes, such as oprJ, mexR, mexA, $n f x B$, ampC, and oprM, in avian $P$. aeruginosa isolates was conducted, and the results revealed the presence of these genes with percentages of $100 \%, 71.4 \%, 85.7 \%, 76.2 \%, 45.2 \%$, and $50 \%$, respectively [38]. Furthermore, the presence of quorum sensing genes in $P$. aeruginosa regulates and provides information about the expression of virulence factors and pathogenicity of the organism [55]. P. aeruginosa quorum sensing genes, such as LasI, LasR, Rh11, and $R h I R$, and other virulence genes that inhibit protein biosynthesis, such as tox $A$, were detected in at prevalences of $85.7 \%, 92.9 \%, 66.7 \%, 80.9 \%$, and $80.9 \%$, respectively [38]. However, other studies detected that the incidence rate of $P$. aeruginosa virulence genes (tox $A$, exoS, lasB, and lasI) was $71.42 \%$ for each of them, while it was $100 \%$ for oprL gene [25].

Moreover, $P$. aeruginosa can acquire resistance by mutation through chromosome-encoded genes or horizontal gene transfers of antibiotic resistance determinants $[18,56]$. It is well known that $P$. aeruginosa harbors antibiotic-resistant plasmids, integrons, and transposons that transfer them to other spp. Rasamiravaka et al. [31] suggested that the opportunistic nature of $P$. aeruginosa, formation of biofilms, and presence of chronic infections may be the causes of multiple drug resistance. Therefore, intrinsic and acquired antibiotic resistance mechanisms cause difficulties in the treatment of $P$. aeruginosa infection [57]. Furthermore, antibiotic-resistant Pseudomonas spp. have been detected in chicken meat [58]. A recent study by Heir et al. [17] proved the occurrence of antibiotic resistance in psychrotrophic Pseudomonas spp. collected from retail chicken in Norway in a 26-year period. Moreover, P. aeruginosa-contaminated chicken carcasses in the abattoir harbor some resistance genes, such as metallo- $\beta$-lactamase $\left(\right.$ bla $_{\mathrm{IMP}-1}, b l a_{\mathrm{IMP}-2}$, bla $_{\mathrm{VIM}-1}$, and bla $\left.{ }_{\mathrm{VIM}-2}\right)$ and AmpC (bla ${ }_{\mathrm{FOX}}$, bla $_{\mathrm{DHA}}$, bla ${ }_{\mathrm{CMY}}$, and bla $\left.{ }_{\mathrm{ACC}}\right)$, which are potential sources for the wide dissemination of antibiotic resistance [52].

Accordingly, both abattoirs and poultry farms are regarded as good grounds for the evolution and spread of antibiotic-resistant $P$. aeruginosa in the non-hospital environment [59]. Moreover, the litter of poultry that contains even a trace number of $P$. aeruginosa organism with antibiotic residues plays an important role in the spread of infection in the environments [38]. The presence of a high resistance pattern by $P$. aeruginosa isolates in poultry meat may be attributed to the continuous use of these antibiotics during the rearing period of birds.

The presence of resistance genes to quaternary ammonium compound disinfectants has also been detected. In $P$. aeruginosa strains from Egyptian chicks' embryos and broiler chickens, the resistance rate to these disinfectants was $14.28 \%$ for qac $A B$ and qacCD genes, while it was $100 \%$ for qacED1 gene [25]. It was detected that genes coding resistance to disinfectants were combined decisively with genes coding for resistance to sulfonamides, trimethoprim, chloramphenicol, aminoglycosides, and $\beta$-lactams [60].

\section{Conclusion}

From the abovementioned, it could be concluded that infection with Pseudomonas spp., especially $P$. aeruginosa, is a disease of significant importance for poultry production and human health. The pathogen is of zoonotic nature, which can be transmitted to humans after handling of poultry carcasses and related products in processing plants and abattoirs. The development of resistance to different antibiotics is common among $P$. aeruginosa strains, in either poultry or humans. Accordingly, strict supervision and application of laws to control antibiotic use in the food chain within the established safe levels should be conducted. 


\section{Authors' Contributions}

WAA collected relevant literature, wrote the original draft, reviewed the manuscript, and approved the final version.

\section{Acknowledgments}

The author did not receive any funds for this study.

\section{Competing Interests} interests.

The author declares that she has no competing

\section{Publisher's Note}

Veterinary World remains neutral with regard to jurisdictional claims in published institutional affiliation.

\section{References}

1. World Health Organization. (2020) WHO Health Topic Page: Zoonoses. World Health Organization, Geneva. Available from: https://www.who.int/topics/zoonoses/en/ Retrieved on 20-07-2020.

2. Grace, D., Mutua, F., Ochungo, P., Kruska, R., Jones, K., Brierley, L., Lapar, L., Said, M., Herrero, M. and Phuc, P.M. (2012) Mapping of poverty and likely zoonoses hotspots. In: Zoonoses Project 4. Report to the UK Department for International Development, International Livestock Research Institute, Nairobi, Kenya.

3. Thompson, A. and Kutz, S. (2019) Introduction to the special issue on emerging zoonoses and wildlife. Int. J. Parasitol. Parasites Wildl., 9: 322.

4. Mena, K.D. and Gerba, C.P. (2009) Risk assessment of Pseudomonas aeruginosa in water. Rev. Environ. Contam. Toxicol., 201: 71-115.

5. Silby, M.W., Winstanley, C., Godfrey, S.A., Levy, S.B. and Jackson, R.W. (2011) Pseudomonas genomes: Diverse and adaptable. FEMS Microbiol. Rev., 35(4): 652-680.

6. Gong, Q., Ruan, M.D., Niu, M.F., Qin, C.L., Hou, Y. and Guo, J.Z. (2018) Immune efficacy of DNA vaccines based on oprL and oprF genes of Pseudomonas aeruginosa in chickens. Poult. Sci., 97(12): 4219-4227.

7. Walker, S.E., Sander, J.E., Cline, J.L. and Helton, J.S. (2002) Characterization of Pseudomonas aeruginosa isolates associated with mortality in broiler chicks. Avian Dis., 46(4): 1045-1050.

8. Fekadu, K. (2010) Pseudomonas infection in chickens. $J$. Vet. Med. Anim. Health, 2(4): 55-58.

9. Handley, J.A., Park, S.H., Kim, S.A. and Ricke, S.C. (2018) Microbiome profiles of commercial broilers through evisceration and immersion chilling during poultry slaughter and the identification of potential indicator microorganisms. Front. Microbiol., 9: 345.

10. Chen, S.H., Fegan, N., Kocharunchitt, C., Bowman, J.P. and Duffy, L.L. (2020) Changes of the bacterial community diversity on chicken carcasses through an Australian poultry processing line. Food Microbiol., 86: 103350.

11. Azam, M.W. and Khan, A.U. (2019) Updates on the pathogenicity status of Pseudomonas aeruginosa. Drug Discov. Today, 24(1): 350-359.

12. Rybtke, M., Hultqvist, L.D., Givskov, M. and TolkerNielsen, T. (2015) Pseudomonas aeruginosa biofilm infections: Community structure, antimicrobial tolerance and immune response. J. Mol. Biol., 427(23): 3628-3645.

13. McEwen, S.A. and Fedorka-Cray, P.J. (2002) Antimicrobial use and resistance in animals. Clin. Infect. Dis., 34(Suppl 3): 93-106.

14. Annan-Prah, A., Agbemafle, E., Asare, P.T. and Akorli, S.Y.
(2012) Antibiotic use, abuse and their public health implication: The contributory role of management flaws in the poultry industry in two agro-ecological zones in Ghana. $J$. Vet. Adv., 2(4): 199-208.

15. Davies, J. and Davies, D. (2010) Origins and evolution of antibiotic resistance. Microbiol. Mol. Biol. Rev., 74(3): 417-433.

16. Maron D.F., Smith, T.J.S. and Nachman, K.E. (2013) Restrictions on antimicrobial use in food animal production: An international regulatory and economic survey. Glob. Health, 9: 48.

17. Heir, E., Moen, B., Åsli, A.W., Sunde, M. and Langsrud, S. (2021) Antibiotic resistance and phylogeny of Pseudomonas spp. Isolated over three decades from chicken meat in the Norwegian food chain. Microorganisms, 9(2): 207.

18. Bonomo, R.A. and Szabo, D. (2006) Mechanisms of multidrug resistance in Acinetobacter species and Pseudomonas aeruginosa. Clin. Infect. Dis., 43(Suppl 2): 49-56.

19. Al-Ahmadi, G.J. and Roodsari, R.Z. (2016) Fast and specific detection of Pseudomonas Aeruginosa from other pseudomonas species by PCR. Ann. Burns Fire Disasters, 29(4): 264-267.

20. El-Gohary, A.H., El-Shehedi M.A. and Safwat, E. (2012) Characterization of Pseudomonas aeruginosa Isolated from Ration and its Viability in the Environment. The $1^{\text {st }}$ Conference of Animal Health Institute Association, Cairo, Egypt, December. p729-737.

21. Kolmos, H.J., Thuesen, B., Nielsen, S.V., Lohmann, M., Kristoffersen, K. and Rosdahl, V.T. (1993) Outbreak of infection in a burns unit due to Pseudomonas aeruginosa originating from contaminated tubing used for irrigation of patients. J. Hosp. Infect., 24(1): 11-21.

22. Qin, X., Emerson, J., Stapp, J., Stapp, L., Abe, P. and Burns, J.L. (2003) Use of real-time PCR with multiple targets to identify Pseudomonas aeruginosa and other non-fermenting gram-negative bacilli from patients with cystic fibrosis. J. Clin. Microbiol., 41(9): 4312-4317.

23. Spilker, T., Coenye, T., Vandamme, P. and Lipuma, J.J. (2004) PCR-based assay for differentiation of Pseudomonas aeruginosa from other Pseudomonas species recovered from cystic fibrosis patients. J. Clin. Microbiol., 42(5): 2074-2079.

24. Eraky, R.D., Abd El-Ghany, W.A. and Soliman, K.M. (2020) Studies on Pseudomonas aeruginosa infection in hatcheries and chicken. J. Hellenic Vet. Med. Soci., 71(1): 1953-1962.

25. Shahat, H.S., Mohamed, H.M.A., Abd Al-Azeem, M.W. and Nasef, S.A. (2019) Molecular detection of some virulence genes in Pseudomonas aeruginosa isolated from chicken embryos and broilers with regard to disinfectant resistance. SVU Inter. J. Vet. Sci., 2(2): 52-70.

26. Mohamed, H.A. (2004) Some studies on Pseudomonas species in chicken embryos and broiler in Assiut governorate. Ass. Univ. Bull. Environ., 7(1): 23-31.

27. Joh, S.J., Kim, M.C., Kwon, Y.K. and Kim, J.H. (2005) Occurrence of Pseudomonas aeruginosa infection in the broilers in Korea. Korean J. Vet. Res., 45(1): 71-74.

28. Van Delden, C.V. (2004) Virulence factors in Pseudomonas aeruginosa. In: Pseudomonas: Virulence and Gene Regulation. Kluwer Academic/Plenum Publishers, New York. p23-45.

29. Fadhil, L., Al-Marzoqi, A.H., Zahraa, M.A. and Shalan, A.A. (2016) Molecular and phenotypic study of virulence genes in a pathogenic strain of Pseudomonas aeruginosa isolated from various clinical origins by PCR: Profiles of genes and toxins. Res. J. Pharm. Biol. Chem. Sci., 7(1): 590-598.

30. Michalska, M. and Wolf, P. (2015) Pseudomonas exotoxin A: Optimized by evolution for effective killing. Front. Microbiol., 6: 963.

31. Rasamiravaka, T., Labtani, Q., Duez, P. and ElJaziri, M. (2015) The Formation of biofilms by Pseudomonas aeruginosa: A review of the natural and synthetic compounds 
interfering with control mechanisms. Biomed. Res. Int., 2015: 759348 .

32. Ertugrul, B.M., Oryasin, E., Lipsky, B.A., Willke, A. and Bozdogan, B. (2018) Virulence genes $f l i C$, toxA and $p h z S$ are common among Pseudomonas aeruginosa isolates from diabetic foot infections. Infect. Dis. (Lond.), 50(4): 273-279.

33. Li, M., Wei, X., Li, Y., Feng, T., Jiang, L., Zhu, H., Yu, X., Tang, J., Chen, G., Zhang, J. and Zhang, X. (2020) PM2.5 in poultry houses synergizes with Pseudomonas aeruginosa to aggravate lung inflammation in mice through the NF- $\mathrm{KB}$ pathway. J. Vet. Sci., 21(3): 46.

34. Kebede, F. (2010) Pseudomonas infection in chickens. $J$. Vet. Med. Anim. Health, 2(4): 55-58.

35. Satish, S. and Priti, M. (2015) Pseudomonas aeruginosa infection in broiler chicks in Jabalpur. Int. J. Ext. Res., 6: 37-39

36. Saif-Edin, M.E. (1983) Some Studies on Pseudomonas Infection in Chickens. M. V. Sc., Thesis, Faculty of Veterinary Medicine, Assiut University, Egypt.

37. Hassan, H.M. (2013) Characterization of Pseudomonas aeruginosa Isolated from Different Pathological Lesions in Chickens. M. V. Sc. Thesis, Faculty of Veterinary Medicine, Beni-Suef University, Egypt.

38. Farghaly, E.M., Roshdy, H., Bakheet, A.A., Abd El-Hafez, S.A. and Badr, H. (2017) Advanced studies on Pseudomonas aeruginosa infection in chicken. Anim. Health Res. J., 5(4): 207-217.

39. Elsayed, M.S.A., Ammar, A.M., Al-Shehri, Z.S., Abd-El Rahman, H. and Abd-El Rahman, N.A. (2016) Virulence repertoire of Pseudomonas aeruginosa from some poultry farms with detection of resistance to various antimicrobials and plant extracts. Cell. Mol. Biol., 62: 124-128.

40. Badr, J.M., El Saidy, F.R. and Abdelfattah, A.A. (2020) Emergence of multidrug resistant Pseudomonas aeruginosa in broiler chicks. Int. J. Microbiol. Biotechnol., 5(2): 41-47.

41. Gellatly, S.L. and Hancock, R.E. (2013) Pseudomonas aeruginosa: New insights into pathogenesis and host defenses. Pathog. Dis., 67(3): 159-173.

42. Vincent, J.L. (2003) Nosocomial infections in adult intensive-care units. Lancet, 361(9374): 2068-2077.

43. Oliver, A., Mena, A. and Macià, M.D. (2008) Evolution of Pseudomonas aeruginosa pathogenicity: From acute to chronic infections. In: Baquero, F., Nombela, C., Cassell, G.H., Gutiérrez, J.A., editors. Evolutionary Biology of Bacterial and Fungal Pathogens. ASM Press, Washington, DC. p433-44.

44. Hinton, A., Cason, J.A. and Ingram, K.D. (2004) Tracking spoilage bacteria in commercial poultry processing and refrigerated storage of poultry carcasses. Int. J. Food Microbiol., 91(2): 155-165.

45. Morales, P.A., Aguirre, J.S., Troncoso, M.R. and Figueroa, G.O. (2016) Phenotypic and genotypic characterization of Pseudomonas spp. present in spoiled poultry fillets sold in retail settings. LWT Food Sci. Technol., 73: 609-614.

46. Møretrø, T. and Langsrud, S. (2017) Residential bacteria on surfaces in the food industry and their implications for food safety and quality. Comp. Rev. Food Sci. Food Saf., 16(5):
1022-1041

47. Quintieri, L., Fanelli, F. and Caputo, L. (2019) Antibiotic resistant Pseudomonas spp. Spoilers in fresh dairy products: An underestimated risk and the control strategies. Foods, 8(9): 372.

48. Harisberger, M., Gobeli, S., Hoop, R., Dewulf, J., Perreten, V. and Regula, G. (2011) Antimicrobial resistance in Swiss laying hens, prevalence and risk factors. Zoonoses Public Health, 58(6): 377-387.

49. Grenni, P., Ancona, V. and Caracciolo, A.B. (2018) Ecological effects of antibiotics on natural ecosystems: A review. Microchem. J., 136(1): 25-39.

50. Hirsch, E.B. and Tam, V.H. (2010) Impact of multidrug resistant Pseudomonas aeruginosa infection on patient outcomes. Expert. Rev. Pharm. Outcomes Res., 10(4): 441-451.

51. Hayford, O. (2017) Isolation and Characterisation of Multidrug Resistant Pseudomonas aeruginosa from Clinical, Environmental and Poultry Litter Sources in Ashanti Region of Ghana. Mv.Sc, Thesis, Kwame Nkrumah University of Science and Technology, Kumasi, Ghana.

52. Ejikeugwu, C., Nworie, O., Saki, M., Al-Dahmoshi, H.O.M., Al-Khafaji, N.S.K., Ezeador, C., Nwakaeze, E., Eze, P., Oni, E., Obi, C., Iroha, I., Esimone, C. and Adikwu, M.U. (2021) Metallo- $\beta$-lactamase and AmpC genes in Escherichia coli, Klebsiella pneumoniae, and Pseudomonas aeruginosa isolates from abattoir and poultry origin in Nigeria. $B M C$ Microbiol., 21(1): 124.

53. Li, X.Z., Nikaido, H. and Poole, K. (1995) Role of mexA-mexBoprMin antibiotic efflux in Pseudomonas aeruginosa. Antimicrob. Agents Chemother., 39(9): 1948-1953.

54. Habeeb, R.H., Saad, S.N. and Al-Jubory, A. (2012) A study of efficacy of disinfectants and bacterial contamination in Alhilla teaching hospital. Med. J. Babylon, 9(4): 890-900.

55. Kumar, R., Chhibber, S., Gupta, V. and Harjai, K. (2011) Screening and profiling of quorum sensing signal molecules in Pseudomonas aeruginosa isolates from catheterized urinary tract infection patients. Indian J. Med. Res., 134(2): 208-213.

56. Breidenstein, E.B., De La Fuente-Nunez, C. and Hancock, R.E. (2011) Pseudomonas aeruginosa: All roads lead to resistance. Trends Microbiol., 19(8): 419-426.

57. Lister, P.D., Wolter, D.J. and Hanson, N.D. (2009) Antibacterial-resistant Pseudomonas aeruginosa: Clinical impact and complex regulation of chromosomally encoded resistance mechanisms. Clin. Microbiol. Rev., 22(4): 582-610.

58. Amos, G.C.A., Carter, A., Hawkey, P.M., Gaze, W.H. and Wellington, E.M. (2015) The hidden resistome of retail chicken meat. J. Glob. Antimicrob. Resist., 3(1): 44-46.

59. Luo, Q., Wang, Y. and Xiao, Y. (2020) Prevalence and transmission of mobilized colistin resistance (MCR) gene in bacteria common to animals and humans. Biosaf. Health, 2(2): 71-78.

60. Schill, F., Abdulmawjood, A., Klein, G. and Reich, F. (2017) Prevalence and characterization of extended-spectrum $\beta$-lactamase (ESBL) and AmpC $\beta$-lactamase producing Enterobacteriaceae in fresh pork meat at processing level in Germany. Int. J. Food Microbiol., 257: 58-66. 